\title{
Craniocervical dystonia questionnaire (CDQ-24): development and validation of a disease-specific quality of life instrument
}

\author{
J Müller, J Wissel, G Kemmler, B Voller, T Bodner, A Schneider, G K Wenning, W Poewe, and the \\ Austrian Botulinum Toxin and Dystonia Study Group
}

J Neurol Neurosurg Psychiatry 2004;75:749-753. doi: 10.1136/jnnp.2003.013441

See end of article for authors' affiliations

Correspondence to:

Prof W Poewe

Department of 'Neurology,

Innsbruck University

Hospital, Anichstr. 35,

A-6020 Innsbruck;

werner.poewe@

vibk.ac.at

Received 3 March 2003

In revised form

25 April 2003

Accepted 4 October 2003

\begin{abstract}
Objective: To develop and test a questionnaire for measuring quality of life in patients with craniocervical dystonia.

Methods: A 29-item pool was developed based on semi-structured interviews of patients with cervical dystonia (CD) and blepharospasm (BSP). This preliminary questionnaire was administered to 203 consecutive patients with CD and BSP from Austrian dystonia and botulinum toxin outpatient clinics. For scale generation, a combination of exploratory factor and cluster analysis was applied. This resulted in the 24-item version of the instrument (CDQ-24) based on five subscales: Stigma, Emotional wellbeing, Pain, Activities of daily living, and Social/family life. The validity and reliability of the CDQ-24 was assessed in 231 consecutive patients with $C D$ and BSP different from those examined with the preliminary questionnaire. This second survey included the CDQ-24, a generic QoL instrument (SF-36) and clinical rating scales. Sensitivity to change was analysed in 51 previously untreated (de novo) patients four weeks and one year following the first botulinum toxin treatment.

Results: Internal consistency reliability was satisfactory for all subscales, with values of Cronbach's $\alpha$ ranging from 0.77 to 0.89 . The CDQ-24 subscales showed moderate to high correlations with those SF-36 subscales measuring similar aspects (Pearson's correlation $r=0.50-0.73 ; p<0.001$, each). Sensitivity to change was confirmed by highly significant improvements of all CDQ-24 subscores in the de novo patients from baseline to four week follow up. One year follow up data revealed a stable improvement.

Conclusion: The CDQ-24 is the first fully validated and disease specific questionnaire to evaluate quality of life of patients with cervical dystonia and blepharospasm and we propose its use in clinical trials as well as in daily clinical practice.
\end{abstract}

\section{INTRODUCTION}

Primary dystonia is one of the most prevalent movement disorders with a minimum prevalence of 98 per 100000 according to a recent population based survey. ${ }^{1}$ Cervical dystonia (CD) and blepharospasm (BSP) are the most common forms and account for about $75 \%$ of cases with primary focal dystonia. ${ }^{2}$ Patients with craniocervical dystonia face a lifetime of chronic visible disability ${ }^{3}$ and previous studies have demonstrated impaired health related quality of life (HR-QoL) in patients with $\mathrm{CD}^{4-9}$ and BSP. ${ }^{8}{ }^{9}$ The generic HR-QoL measures used in these studies consistently demonstrated lower physical and mental QoL scores in craniocervical dystonia compared with healthy controls. While generic QoL instruments compare outcomes across different populations they do not measure specific problems of diagnostic groups. ${ }^{10}$ A recently published study revealed that QoL in patients with CD is mainly predicted by factors such as self esteem, self deprecation, social participation, social support, stigma, anxiety, and depression in addition to disease severity and response to botulinum toxin $^{11}$-all of which are not addressed by a generic QoL instrument.

The purpose of this study was to develop a disease specific instrument to measure the QoL of patients with craniocervical dystonia and to test its reliability and validity. The instrument was to be particularly relevant to patients with craniocervical dystonia and sensitive to assess functional health and treatment effects in clinical trials and in daily clinical practice.

\section{METHODS}

A three stage strategy was used to develop and test the 24item Craniocervical Dystonia Questionnaire (CDQ-24) according to Peto et al. ${ }^{12}$

\section{Stage I: Item generation}

Exploratory semi-structured interviews were carried out with 20 patients ( $C D, n=10$; $B S P, n=10$ ) by a neuropsychologist (TB). Patients were asked to indicate relevant aspects of life adversely affected by dystonia. Based on these data, a team of neurologists and psychologists developed a preliminary 29item questionnaire.

\section{Stage II: Item reduction and scale generation}

The preliminary 29-item questionnaire was administered to 203 consecutive patients (mean (SD) age 56 (12), 36\% male) with CD (56\%) and BSP (44\%) from Austrian dystonia and botulinum toxin outpatient clinics (see appendix 1). For scale generation, a combination of exploratory factor analysis (principal components method with varimax rotation) and cluster analysis was applied. As the factor analysis solutions studied $(4,5$, and 6 factors, as suggested by the "scree" criterion $)^{13}$ gave rise to a very large first factor, cluster analysis (K-means item clustering) was employed to arrive at a more meaningful and tractable set of subscales. This

Abbreviations: ADL, activities of daily living; BSP, blepharospasm; BTX, botulinum toxin; $C D$, cervical dystonia; $H R-Q o L$, health related quality of life; SF-36, Short Form-36 Health Survey 
resulted in a scale with five domains: stigma (nine items), emotional wellbeing (five items), pain (three items), activities of daily living (ADL; seven items), and social/family life (four items) - one item did not match with any of the five domains. Internal consistency was satisfactory for all preliminary subscales $(\alpha>0.7$ each). In the subsequent item reduction phase, five of the original items that met at least one of the following criteria were omitted: (i) strong ceiling and floor effects, (ii) low internal consistency with the other items of a subscale, or (iiI) redundancy within a subscale. This resulted in the final 24-item version of the instrument (CDQ-24) (see appendix 1) based on five subscales:

- Stigma (questions 7, 8, 9, 10, 18, 22)

- Emotional wellbeing (questions 11, 12, 13, 14, 15)

- Pain (questions 4, 5, 21)

- $\mathrm{ADL}$ (questions 1, 2, 3, 6, 19, 20)

- Social/family life (questions 16, 17, 23, 24)

Each item consists of five statements representing increasing severity of impairment, scored from 0 to 4 . Subjects were instructed to indicate how they felt during the past two weeks because of dystonia by selecting one of the five statements for each item. In order to obtain comparable scores for the individual subscales, the raw subscores (= sum of the individual item scores) were linearly transformed to a 0-100 scale, where a score of 0 indicates the best and a score of 100 the worst possible QoL.

\section{Stage III: Testing the CDQ-24}

The validity and reliability of the CDQ- 24 was assessed in a second study of 231 consecutive patients with cranial and cervical dystonia (table 1) different from the patients assessed in Stage II. This second survey included the CDQ24, a generic QoL instrument (Short Form-36 Health Survey, SF-36) ${ }^{14}$ and clinical rating scales for CD and BSP. The severity of CD was assessed by the Tsui scale, which measures the severity and duration of head deviation, shoulder elevation, and head tremor/jerks. ${ }^{15}$ In addition, neck pain was rated on a four point scale (absent, mild, moderate, severe). ${ }^{16}$ The severity of BSP was assessed by the treating physician on a global impression scale $(0-4) .{ }^{17}$

\section{Reliability properties}

The internal consistency of the subscales was quantified by Cronbach's $\alpha$ in the total sample of 231 patients. Test-retest reliability was evaluated in 51 previously untreated (de novo) patients by intraclass correlation coefficients.

\section{Construct validity}

The convergent validity of the CDQ-24 with the SF-36 was assessed in all 231 patients by investigating Pearson's correlation matrix CDQ- $24 \times \mathrm{SF}-36$. We hypothesised that correlations between corresponding subscales of the two instruments would be at least moderately high ( $r$ 0.5-0.7), while correlations between not well matching pairs of subscales would be lower. ${ }^{18}$ Discriminant validity was assessed by relating CDQ-24 subscales to clinical scoresfor example, Tsui score. Due to the ordinal structure of these variables Spearman's rank correlations were used. In order to check the dimensional structure of the CDQ-24 obtained in the item reduction and scale generation stage (II), a factor analysis (principal components method, varimax rotation) was performed.

Sensitivity to change (four weeks and one year data) Sensitivity to change was analysed in all de novo patients four weeks following the first botulinum toxin (BTX) injection by determining the effect size of the change between baseline and post-treatment CDQ-24 scores, defined as the difference of baseline and post-treatment means divided by the standard deviation at baseline. ${ }^{19}$ In addition, sensitivity to change was assessed after one year of repetitive three monthly BTX injections in all de novo patients.

\section{RESULTS}

A total of 231 consecutive patients with CD $(60 \%)$ and BSP $(40 \%)$ were included in the third phase of the study (Stage III). Patient characteristics are shown in table 1. Except for age, there were no significant differences in patient characteristics between CD and BSP. Fifty one patients with craniocervical dystonia (22\%) were newly diagnosed (de novo) and had never received BTX treatment before.

\section{Descriptive statistics}

Descriptive statistics of the CDQ-24 subscales assessed at baseline are displayed in table 2, both for the total sample and for CD and BSP separately. Patients with BSP showed better HR-QoL (lower scores) in the subscales for "pain" $(\mathrm{p}<0.001)$ and "stigma" $(\mathrm{p}=0.01)$ compared with patients with $\mathrm{CD}$. There were no relevant ceiling effects and a considerable floor effect was only observed in one domain (social/family life).

\section{Reliability properties}

Internal consistency reliability was satisfactory for all subscales, with values of Cronbach's $\alpha$ ranging from 0.77 to 0.89 (table 2). Test-retest reliability was generally high;

Table 1 Patient characteristics of the sample $(n=231)$ used to assess reliability and validity of the CDQ-24 (Stage III)

\begin{tabular}{lll}
\hline Patient characteristics & Cervical dystonia & Blepharospasm \\
\hline Number & 139 & 92 \\
Sex (\% women) & 64.7 & 60.9 \\
Mean (SD) age (years)* & $53(12)$ & $64(10)$ \\
Mean (SD) disease duration (years) & $8.0(5.9)$ & $6.9(6.8)$ \\
Severity of dystonia & Tsui score (mean \pm SD) & Global impression score:0-4 (mean \pm SD) \\
& $6.7(3.7)$ & $2.3(0.9)$ \\
Neck pain (\%) & 67.1 & - \\
Additional dystonic features (\%) & Segmental dystonia: 5.8 & Meige's syndrome: 28.3 \\
De novo patients (\%)† & 22.3 & 21.7 \\
Married (\%) & 74.8 & 74.4 \\
Employment rate (\% of patients & $50.0(n=98)$ & $57.1(n=35)$ \\
$\leqslant 60$ years only) & &
\end{tabular}

${ }^{*} \mathrm{p}=0.001$, cervical dystonia $v$ blepharospasm.

†No previous botulinum toxin treatment.

$\mathrm{SD}$, standard deviation. 
Table 2 Descriptive statistics, ceiling effects and internal consistency reliability of CDQ-24 subscales in Stage III of the study

\begin{tabular}{|c|c|c|c|c|c|c|}
\hline $\begin{array}{l}\text { CDQ-24 subscale } \\
\text { (no. of items) }\end{array}$ & $\begin{array}{l}\text { Cervical dystonia } \\
\text { ( } n=139 \text { ) } \\
\text { Mean (SD) }\end{array}$ & $\begin{array}{l}\text { Blepharospasm } \\
\text { (n=92) } \\
\text { Mean (SD) }\end{array}$ & $\begin{array}{l}\text { Total sample } \\
(n=231) \\
\text { Mean (SD) }\end{array}$ & $\begin{array}{l}\text { \% scoring min. } \\
(0) \dagger\end{array}$ & $\begin{array}{l}\text { \% scoring max. } \\
(100) \dagger\end{array}$ & $\begin{array}{l}\text { Internal consistency } \\
\text { (Cronbach's } \alpha \text { ) }\end{array}$ \\
\hline Stigma (6) & $45.6(26.4)$ & $36.3(23.2)^{*}$ & $41.9(25.5)$ & 2.2 & 1.3 & 0.89 \\
\hline Emotional wellbeing (5) & $36.2(24.3)$ & $32.4(24.5)$ & $34.7(24.4)$ & 9.1 & 0.9 & 0.88 \\
\hline Pain (3) & $44.1(27.0)$ & $24.5(24.5)^{*}$ & $36.3(27.6)$ & 17.8 & 1.7 & 0.79 \\
\hline Activities of daily living (6) & $38.3(23.1)$ & $43.3(23.0)$ & $40.3(23.1)$ & 3.0 & 0.4 & 0.77 \\
\hline Social/family life (4) & $22.7(23.7)$ & 15.5 (19.4) & $19.8(22.3)$ & 34.8 & 0.4 & 0.85 \\
\hline Total score (24) & $37.8(20.4)$ & $32.3(18.2)$ & $35.6(19.7)$ & 0.0 & 0.0 & 0.94 \\
\hline
\end{tabular}

${ }^{*} \mathrm{p}<0.01$ cervical dystonia $v$ blepharospasm (Mann-Whitney $U$ test)

t0, best quality of life; 100, worst quality of life.

intraclass correlation coefficients reached values of 0.9 or higher for all domains.

\section{Construct validity}

Analysis for convergent validity showed moderate to high correlations between those CDQ-24 and SF-36 subscales assumed to measure similar aspects. Thus, the CDQ-24 "emotional wellbeing" subscale correlated most strongly with SF-36 "mental health" (Pearson's correlation coefficient $r=0.70, \mathrm{p}<0.001$ ), the CDQ-24 "pain" subscale with the SF-36 "bodily pain" domain $(r=0.73, \mathrm{p}<0.001)$, and the CDQ-24 "social/family life" subscale with SF-36 "social functioning" $(r=0.60, \mathrm{p}<0.001)$. The CDQ-24 subscale on "ADL" showed moderately high correlations $(r=0.50-0.55$, $\mathrm{p}<0.01$, each) with those SF-36 subscales addressing physical functioning (including "physical functioning", "role physical" and "vitality"). The CDQ-24 "stigma" subscale (which has no direct SF-36 equivalent) showed its highest correlation with the SF-36 "social functioning domain" $(\mathrm{r}=0.57, \mathrm{p}<0.001)$. The correlations of CDQ-24 subscales with those SF-36 subscales measuring different aspects were consistently lower than those given above, which is in accordance with the hypothesised correlation pattern.

\section{Discriminant validity}

Patients with CD showed low correlations of the CDQ-24 subscores with the Tsui-score, attaining statistical significance for "ADL" $(r=0.29, \mathrm{p}=0.01)$, "social/family life" $(r=0.19, \quad \mathrm{p}=0.04), \quad$ "emotional well-being" $\quad(r=0.18$, $\mathrm{p}=0.04)$. Correlations of CDQ-24 subscores with pain ratings were higher and reached statistical significance for all CDQ24 subscales $(\mathrm{p}<0.05$, each) and for the CDQ-24 total score $(r=0.48, \mathrm{p}<0.001)$ in $\mathrm{CD}$. In patients with BSP, correlations of the CDQ-24 subscores with the clinical global impression score were also low, attaining statistical significance for two of the five CDQ-24 subscales (ADL $r=0.31, p=0.003$; emotional wellbeing $r=0.2 \mathrm{l}, \mathrm{p}=0.047)$ and the CDQ-24 total score $(r=0.25, \mathrm{p}=0.019)$.

\section{Dimensional structure of the CDQ-24}

A factor analysis performed on all patients who completed the CDQ-24 gave rise to a five factor model according to the eigenvalue criterion (cut-off value 1); this model explained $67.9 \%$ of the total variance. With only two exceptions (two items of the ADL subscale) the allocation of all 24 items to the factors-as suggested by their loadings-coincided with the previously defined domain structure of the instrument. The single two items with suboptimal assignment to the ADL subscale showed fairly high side loadings $(r=0.38$ and 0.39$)$ on the factor ADL. Overall, a confirmation of the hypothesised domain structure of the instrument was obtained.

Sensitivity to change (four weeks and one year data) The CDQ-24 scores at baseline and four weeks following the first BTX treatment in de novo patients are shown in table 3; one year follow up data for 47 of the 51 (92\%) de novo patients are shown in fig 1 . The largest treatment effect was observed in the "stigma" subscale with an effect size of 0.72 at four week follow-up. Patients with CD improved in all CDQ-24 subscales following BTX treatment while those with cranial dystonia showed a significant improvement in all but one subscale (pain). One year follow up data revealed a stable improvement with significantly improved CDQ-24 total scores compared with baseline and to the first follow up at four weeks ( $p<0.001$, each; see fig 1$)$. A weak correlation was identified between changes in the physicians' rating scale and the CDQ-24 total score in BSP $(r=0.3, \mathrm{p}=0.1)$ four weeks post-BTX. Otherwise, none of the changes of CDQ-24 scores were related to changes in physicians' ratings following treatment.

\section{DISCUSSION}

The CDQ-24 was developed as a disease specific QoL questionnaire that addresses the perceptions and concerns of patients with craniocervical dystonia including CD and BSP. Our results indicate that the CDQ-24 meets important clinimetric requirements such as reliability, validity, and sensitivity to change. We therefore propose its use in clinical trials as well as in daily clinical practice.

The content of the questionnaire addresses the special concerns of patients with craniocervical dystonia and includes all the relevant determinants of QoL in people with $\mathrm{CD}$ as recently established by the Epidemiological Study of Dystonia in Europe (ESDE) collaborative group. ${ }^{11}$ Convergent validity was assessed in a large sample of 231 consecutive patients with $\mathrm{CD}$ and BSP by means of correlations of subscales of the CDQ-24 with those subscales of the SF-36 that measure related experiences. As outlined above, all CDQ24 subscales demonstrate good convergent validity for patients with craniocervical dystonia.

The SF-36 is a generic HR-QoL measure that has undergone considerable amount of testing for reliability and validity, $^{2021}$ and previous studies have demonstrated impaired HR-QoL in patients with craniocervical dystonia using the SF-36. ${ }^{8}$ However, these studies indicate that the therapeutic efficacy of BTX therapy is only partially reflected by the SF-36. Apparently, generic scales such as the SF-36 can only provide information on the impact of the disease in relation to published norms. The CDQ-24 on the other hand can be used to evaluate the impact of the disease on areas not covered by generic measures which are of considerable concern to patients with craniocervical dystonia.

The CDQ-24 also showed good reliability properties. For both reliability measures used, internal consistency and testretest reliability, the criteria for good scale performance were satisfied by all subscales of the instrument. ${ }^{22}$

The sensitivity to change of the CDQ-24 was examined in a large group of "de novo" patients with craniocervical dystonia who had never received BTX before. These de novo patients were reassessed four weeks and one year following 


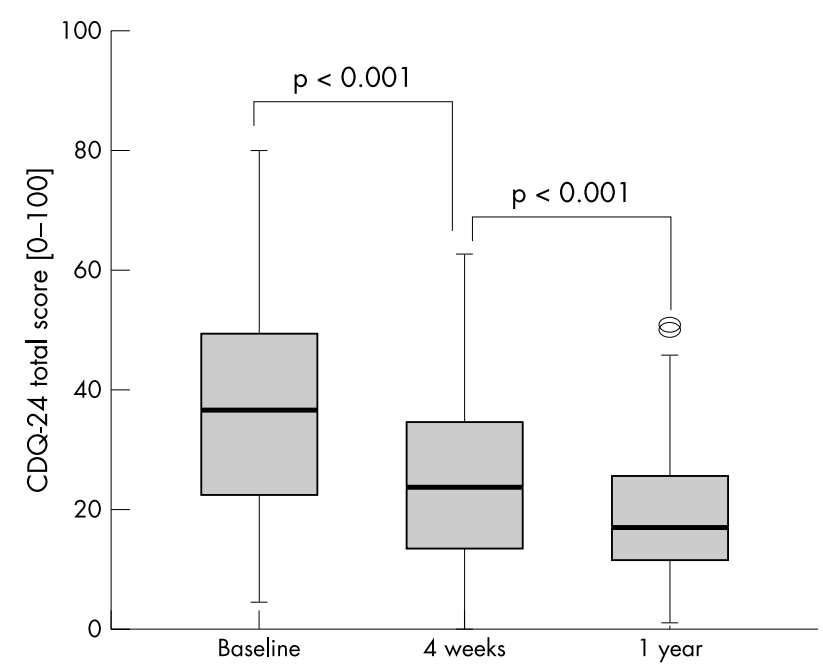

Figure 1 Temporal course of the CDQ-24 in 47 previously untreated patients with cranial and cervical dystonia at baseline, four weeks following the first botulinum toxin injection and after one year of regular three monthly botulinum toxin treatment. The horizontal lines indicate median values; boxes, 25-75th percentile; error bars, lowest and highest values within 1.5 times the values observed in the percentile boxes; and circles, single cases exceeding 1.5 times the value observed in the percentile boxes.

regular BTX treatment and demonstrated highly significant improvements of the CDQ-24 total score at both times (see table 3 and fig 1). The largest effect size was obtained in the stigma subscale. Some or severe perceived stigma is present in the majority of patients with cervical dystonia and was found to severely affect social, private and working lives. ${ }^{23}$ Notably, no correlations between changes in the stigma or any other CDQ-24 subscale and changes of clinical rating scales were observed at both follow up investigations (four weeks and 12 months). These results indicate that the CDQ24 is able to capture changes in patients' wellbeing which are not reflected in clinical rating scales of dystonia severity. In addition, the present study provides evidence for excellent responsiveness of the CDQ-24 to treatment induced changes, which appears to be superior to generic instruments like the SF-36. ${ }^{9}$

In conclusion, the CDQ-24 is an appropriate, validated and useful tool to assess QoL in clinical trials and in daily clinical practice. The CDQ-24 appears to be sensitive to changes that matter to patients but are not the primary focus of clinicians'

Table 3 Sensitivity to change four weeks following the first botulinum toxin treatment in de novo patients $(n=51)$ with craniocervical dystonia

\begin{tabular}{lllll}
\hline & $\begin{array}{l}\text { Baseline } \\
\text { score } \\
\text { Mean (SD) }\end{array}$ & $\begin{array}{l}\text { Score at four } \\
\text { weeks } \\
\text { Mean (SD) }\end{array}$ & $\begin{array}{l}\text { Effect } \\
\text { size }\end{array}$ & p value* \\
CDQ-24 subscale & $44.7(24.2)$ & $27.3(19.6)$ & 0.72 & $<0.001$ \\
\hline Stigma & $38.3(25.7)$ & $27.2(22.5)$ & 0.43 & $<0.001$ \\
$\begin{array}{l}\text { Emotional wellbeing } \\
\text { Pain }\end{array}$ & $39.1(26.8)$ & $26.3(23.4)$ & 0.47 & $<0.001$ \\
$\begin{array}{l}\text { Activities of daily } \\
\text { living }\end{array}$ & $45.5(24.7)$ & $32.5(23.3)$ & 0.53 & $<0.001$ \\
$\begin{array}{l}\text { Social/family life } \\
\text { Total score all }\end{array}$ & $21.0(23.8)$ & $11.4(15.3)$ & 0.40 & 0.002 \\
patients & $38.9(19.9)$ & $25.8(16.7)$ & 0.67 & $<0.001$ \\
$\quad \begin{array}{lllll}\text { Cranial dystonia } \\
\text { (n=20) }\end{array}$ & $41.2(17.4)$ & $28.3(17.6)$ & 0.73 & 0.01 \\
$\begin{array}{l}\text { Cervical dystonia } \\
\text { (n=31) }\end{array}$ & $37.4(21.5)$ & $24.1(16.2)$ & 0.62 & $<0.001$ \\
\hline *Baseline $v$ 4 weeks: Wilcoxon's matched-pairs test. & \\
SD, standard deviation. & & & \\
\hline
\end{tabular}

assessment which makes the instrument an important addition to clinical outcome measures.

\section{ACKNOWLEDGEMENT}

The authors thank K Schneider for secretarial assistance.

\section{Authors' affiliations \\ J Müller, J Wissel, G K Wenning, W Poewe, University Hospital of \\ Neurology, Innsbruck, Austria \\ G Kemmler, T Bodner, University Hospital of Psychiatry, Innsbruck, Austria \\ B Voller, University Hospital of Neurology, Vienna, Austria \\ A Schneider, Department of Neurology, Hospital BhB Linz, Austria \\ Competing interests: none declared}

CDQ-24: The validated German version of the CDQ-24 can be obtained from the authors. The translated English version of the CDQ-24 is attached (appendix 1) and currently under evaluation.

\section{APPENDIX 1 CRANIOCERVICAL DYSTONIA QUESTIONNAIRE (CDQ-24) -ENGLISH VERSION}

\section{BECAUSE OF DYSTONIA, HOW OFTEN HAVE YOU EXPERIENCED THE FOLLOWING IN THE PAST TWO WEEKS}

Please mark one answer per question and try to answer each question.

1. Have you had problems reading or watching TV?

- never/occasionally/sometimes/often/always

2. Has it been difficult for you to do things you like to dofor example, leisure activities?

- never/occasionally/sometimes/often/always

3. Has it been difficult for you to control the symptoms of Dystonia when you were nervous or under stress?

- never/occasionally/sometimes/often/always

4. Have you suffered from pain/a burning sensation in the face, head, or neck region?

- never/occasionally/sometimes/often/always

5. Have you been prevented from falling asleep by pain or a pulling sensation?

- never/occasionally/sometimes/often/always

6. Has it been difficult for you to do fine work with your fingers-for example, writing, threading a needle?

- never/occasionally/sometimes/often/always

7. Have you avoided situations where many people were present-for example, social events?

- never/occasionally/sometimes/often/always

8. Has your Dystonia made you feel uneasy in public?

- never/occasionally/sometimes/often/always

9. Have you felt the need to conceal your Dystonia from other people?

- never/occasionally/sometimes/often/always

10. Have you worried about how other people react to you?

- never/occasionally/sometimes/often/always 
11. Have you worried about your future?

- never/occasionally/sometimes/often/always

12. Have you felt afraid?

- never/occasionally/sometimes/often/always

13. Have you felt down or depressed?

- never/occasionally/sometimes/often/always

14. Have you been sad or on the verge of tears?

- never/occasionally/sometimes/often/always

15. Have you felt annoyed or bitter?

- never/occasionally/sometimes/often/always

16. Have you felt isolated or lonely because of your Dystonia?

- never/occasionally/sometimes/often/always

17. Have you had problems with close friends or your family due to your Dystonia?

- never/occasionally/sometimes/often/always

18. Have you felt unsure or tense with new people?

- never/occasionally/sometimes/often/always

19. Has it been difficult for you to keep up with the demands of your job or home life?

- not at all/slightly/moderately/severely/very severely

- Employed: yes/no

20. Have you experienced difficulty as a motorist or pedestrian?

- not at all/slightly/moderately/severely/very severely

21. Have you felt hindered by pain/a burning sensation in the face, head or neck region?

- not at all/slightly/moderately/severely/very severely

22. Have you felt you didn't look so good?

- not at all/slightly/moderately/severely/very severely

23. Has your Dystonia had a negative effect on your family life?

- not at all/slightly/moderately/severely/very severely

24. Has your Dystonia negatively affected the relationship with your partner?

- not at all/slightly/moderately/severely/very severely

- Partner: yes/no

\section{APPENDIX 2}

The Austrian Botulinum Toxin and Dystonia Study Group: G Albrecht, E Auff, J Diez, T Entner, I Fuchs, K Fheodoroff, J Grossmann, C Hauert, I Hess-Eberle, M S Hiller, F S Höger, P Hollosi, C N Homann, M Kainer, M Kofler, J Müller, W Poewe, H Rieck, E Rohringer, P Schöggl, A Schneider, P Schnider, C Stadler, E Staudacher, B Voller, V Weiser, K Wenzel, J Wissel.

\section{REFERENCES}

1 Mueller J, Kiechl S, Wenning GK, et al. The prevalence of primary dystonia in the general community. Neurology 2002;59:941-3.

2 The Epidemiological Study of Dystonia in Europe (ESDE) Collaborative Group. A prevalence study of primary dystonia in eight European countries. J Neurol 2000;247:787-92.

3 Marsden CD. Psychogenic problems associated with dystonia. In: Weiner WJ, Lang $A E$, eds. Behavioral Neurology of Movement Disorders. Advances in Neurology, Vol. 65. New York: Raven Press, 1995:319-26.

4 Odergren T, Tollback A, Borg J. Efficacy of botulinum toxin for cervical dystonia. A comparison of methods for evaluation. Scand J Rehabil Med 1994;26:191-5.

5 Gudex CM, Hawthorne MR, Butler AG, et al. Effect of dystonia and botulinum toxin treatment on health-related quality of life. Mov Disord 1998; 13:941-6.

6 Lindeboom R, Brans JW, Aramideh M, et al. Treatment of cervical dystonia: a comparison of measures for outcome assessment. Mov Disord 1998;13:706-12.

7 Brans JW, Lindeboom R, Aramideh M, et al. Long-term effect of botulinum toxin on impairment and functional health in cervical dystonia. Neurology 1998;50:1461-3.

8 Hilker R, Schischniaschvili M, Ghaemi M, et al. Health related quality of life is improved by botulinum neurotoxin type $A$ in long term treated patients with focal dystonia. J Neurol Neurosurg Psychiatry $2001 ; 71: 193-9$.

9 Mueller J, Kemmler G, Wissel J, et al. The impact of blepharospasm and cervical dystonia on health-related quality of life and depression. J Neurol 2002;249:842-6.

10 Patrick DL, Deyo RA. Generic and disease-specific measures in assessing health status and quality of life. Med Care 1989;27(Suppl 3): 217-32.

11 Ben-Shlomo Y, Camfield L, Warner T, ESDE collaborative group. What are the determinants of quality of life in people with cervical dystonia. J Neurol Neurosurg Psychiatry 2002;72:608-14.

12 Peto V, Jenkinson C, Fitzpatrick R. PDQ-39: a review of the development, validation and application of a Parkinson's disease quality of life questionnaire and its associated measures. J Neurol 1998;245:S10-14.

13 Dillon WR, Goldstein M. Multivariate Analysis. New York: Wiley, 1984.

14 Ware JE. The SF-36 health survey. In: Spilker B, ed. Quality of life and pharmacoeconomics in clinical trials, 2nd edn. Philadelphia: Lippincott-Raven, 1996:337-45.

15 Tsui JK, Eisen A, Stoessl AJ, et al. Double-blind study of botulinum toxin in spasmodic torticollis. Lancet 1986;2:245-7.

16 Poewe W, Deuschl G, Nebe A, et al. What is the optimal dose of botulinum toxin $A$ in the treatment of cervical dystonia? Results of a double blind, placebo controlled, dose ranging study using Dysport ${ }^{\mathbb{Q}}$. I Neurol Neurosurg Psychiatry 1998;64:13-17.

17 Jankovic J, Schwartz K, Donovan DT. Botulinum toxin treatment of cranialcervical dystonia, spasmodic dysphonia, other focal dystonias and hemifacial spasm. J Neurol Neurosurg Psychiatry 1990;53:633-9.

18 Streiner DL, Norman GR. Health measurement scales, 2nd edn. Oxford: Oxford Medical Publications, 1995.

19 Kazis LE, Anderson JJ, Meenan RF. Effect sizes for interpreting changes in health status. Med Care 1989;27:S178-89.

20 Ware JE, Gandek B. Overview of the SF-36 health survey and the international quality of life assessment (IQOLA) project. $J$ Clin Epidemiol 1998;51:903-12.

21 Jenkinson C, Wright L, Coulter A. Criterion validity and reliability of the SF-36 in a population sample. Qual Life Res 1994;3:7-12.

22 Fayers PM, Machin D. Quality of life. Assessment, analysis and interpretation. Chichester: Wiley \& Sons, 2000.

23 Papathanasiou I, MacDonald L, Whurr R, et al. Perceived stigma in spasmodic torticollis. Mov Disord 2001;16:280-5. 